\title{
Niveles de ansiedad en judokas participantes en el 410 Campeonato Militar de Judo Interejércitos 2017
}

\author{
Roberto RUIZ-BARQUÍN¹, José Juan ROBLES PÉREZ², Santiago GARCÍA ESTEBARANZ³ \\ ${ }^{1}$ Universidad Autónoma de Madrid (España) \\ ${ }^{2}$ Escuela Central de Educación Física, Escuela de Guerra, Ejército de Tierra Español (España) \\ ${ }^{3}$ Servicio de Psicología. Dirección General de la Guardia Civil (España)
}

\author{
I Congreso Nacional de Entrenadores de Judo, Murcia (España), 13-15 de julio, 2018
}

\begin{abstract}
Resumen
Los objetivos del estudio fueron: describir los niveles de ansiedad en judokas de competición participantes en el $41^{\text {o }}$ Campeonato Militar de Judo Interjércitos del año 2017; establecer posibles diferencias en los niveles de ansiedad hallados en función del sexo y el peso; y establecer posibles relaciones entre los niveles de ansiedad hallados y el puesto ocupado en campeonato individual. La muestra final estuvo compuesta por 28 judokas (18 hombres y 10 mujeres) pertenecientes a dos equipos participantes en el Campeonato (Ejército de Tierra y Guardia Civil), siendo la edad media de la muestra de 34.29 años $(D T=5.12)$. De forma previa a la competición se administró el cuestionario de ansiedad ISRA-B versión Respuestas. Los resultados mostraron que los judokas evaluados presentaron menores niveles de ansiedad cognitiva $(p<.05)$, ansiedad fisiológica $(p<.001)$ y ansiedad motora $(p<.001)$ al compararlos con otras muestras de deportistas. Asimismo, se hallaron diferencias significativas en los niveles ansiedad en función del sexo, obteniendo mayores niveles de ansiedad motora el grupo de mujeres $(p<.05)$. En función del peso sólo se mostraron resultados significativos en judokas masculinos $(p<.05)$, donde los más pesados presentaron mayores niveles de ansiedad cognitiva $(p<.05)$. Sólo se hallaron correlaciones significativas entre los niveles de ansiedad motora y el puesto obtenido en competición en el grupo masculino ( $r h o=-.635 ; p<.05)$. Los resultados confirman la necesidad de realizar intervenciones psicológicas diferenciales considerando las variables sexo y categoría de peso.
\end{abstract}

Palabras clave: Judo; ansiedad; Campeonato de Judo Militar Interejércitos; Ejército de Tierra; Guardia Civil.

\begin{abstract}
Anxiety levels in judokas participating in the 41th Inter-Army Military Championship of Judo 2017
\section{Abstract}

The aims of this study were: to describe the anxiety levels of competitive judokas who participated in the 41st Inter-army Military Championship of Judo, year 2017; to detect differences in anxiety levels depending on sex and weight categories; to explore if there was a relation between the anxiety levels and the judokas' final results at the tournament. The sample was composed of 28 judokas (18 men and 10 women) belonging to two teams (Army and Guardia Civil), having an average age of 34.29 years $(S D=5.12)$. Before competition, the ISRA-B questionnaire, answers version, was administered. The results showed that judokas had lower levels of cognitive anxiety $(p<.05)$, physiological anxiety $(p<.001)$ and motor anxiety $(p<.001)$ than other samples of athletes. Likewise, there were significant differences in anxiety levels according to sex, having women higher levels of motor anxiety in $(p<.05)$. In terms of weight, only significant results were found in male judokas $(p<.05)$, where the heavier judokas had higher levels of cognitive anxiety $(p<.05)$. Only significant correlations were found between motor anxiety levels and the position obtained in competition in the male group (rho = $.635, p<.05)$. The results confirmed the necessity to perform differential psychological interventions considering the sex and weight category variables.
\end{abstract}

Keywords: Judo; Anxiety; Inter-Army Military Judo Championships; Army; Guardia Civil.

\section{Introducción}

Simon y Martens (1977) ya destacaban cómo los deportes de contacto manifiestan elevados niveles de ansiedad cognitiva y una menor autoconfianza, debido en parte a la amenaza que provoca la confrontación personal en el combate. Desde una perspectiva psicológica, la evaluación de la ansiedad puede realizarse desde una perspectiva más rasguista (personalidad) o de estados transitorios y circunscritos a una determina situación momento (Spielberger, Gorsuch, \& Lushene, 1968). A su vez, la evaluación de la ansiedad en deportistas puede realizarse utilizando cuestionarios tradicionales provenientes de la Psicología General (e.g., cuestionario de ansiedad

*Email: Uroberto.ruiz@uam.esU

(C) 2018 Universidad de León. www.unileon.es 
ISRA, Miguel-Tobal \& Cano-Vindel, 2002), o con instrumentos específicos de ansiedad adaptados al ámbito deportivo (e.g., CSAI - Competitive State Anxiety Inventory, de Martens, Vealey, \& Burton, 1990; CSAI-2R, de Andrade, Lois, \& Arce, 2007; Montero, Moreno, González, Pulido, \& Cervelló, 2012; Ruiz, 2009). En el deporte de judo a nivel competitivo es importante analizar las posibles diferencias individuales existentes en los niveles de ansiedad en función de la categoría de sexo, categoría de peso y rendimiento deportivo. Por ello, los objetivos del presente estudio fueron:

(1) Describir los niveles de ansiedad en judokas de competición participantes en el Campeonato Interejércitos Militar de Judo del año 2017;

(2) Establecer posibles diferencias en los niveles de ansiedad hallados en función del sexo y el peso; $y$

(3) Establecer posibles relaciones entre los niveles de ansiedad hallados y el puesto ocupado en campeonato individual.

\section{Método}

\subsection{Participantes}

Participaron en el estudio un total de 30 deportistas, si bien se incluyeron finalmente 28 judokas (13 pertenecientes al equipo de Judo del Ejércitos de Tierra, el 46.4\%, y 15 al equipo de la Guardia Civil, el 53.6\%) debido a que en dos casos las pruebas realizadas estaban incompletas. La edad media de la muestra total fue de 34.29 años $(D T=5.12)$ y el número de horas de entrenamiento al judo dedicados semanalmente fue de 2.81 horas $(D T=1.76)$.

\subsection{Instrumento}

Inventario de Situaciones y Respuestas de Ansiedad Breve - ISRA-B, versión reducida del ISRA en su versión Respuestas (Tobal \& Cano-Vindel, 2002). La utilización de este cuestionario, sólo utilizando la versión Respuestas de Ansiedad, ya ha sido utilizada en el contexto deportivo en fútbol (García-Naveira \& Ruiz-Barquín, 2015). El test estuvo compuesto por 24 ítems y tres factores: manifestaciones de ansiedad a nivel cognitivo (AC), fisiológico (AF) y motor (AM). El formato de respuesta fue una escala de cinco niveles en función de la frecuencia de aparición (desde 0 que es la menor frecuencia de aparición hasta 4 que es la mayor frecuencia de aparición).

\subsection{Procedimiento}

Se informó de los objetivos del estudio a los responsables de los dos equipos participantes en el estudio. Se administraron los correspondientes consentimientos informados y se informó de la voluntariedad de participar en la investigación. La administración de la batería completa de test tuvo una duración aproximada de 20 minutos, y se realizó de forma previa a la competición.

\subsection{Análisis de datos}

Se utilizó el paquete estadístico SPSS 22.0, y se realizaron los siguientes análisis de datos: medias y desviaciones típicas, análisis de frecuencias, prueba de normalidad de Shapiro-Wilk, análisis de diferencia de medias para dos muestras independientes ( $U$ de Mann-Whitney) y análisis de correlaciones mediante el coeficiente Rho de Spearman.

\section{Resultados}

Los niveles de ansiedad hallados mostraron valores inferiores a otras muestras de deportistas (AC, $p<05$; AS y AM, $p<.001$ ). Se hallaron diferencias significativas en ansiedad en función del sexo, donde se obtuvieron mayores niveles de ansiedad motora en el grupo de mujeres $(p<.05)$. En función del peso, sólo se mostraron resultados significativos en judokas masculinos 
$(p<.05)$, donde los más pesados presentaron mayores niveles de ansiedad cognitiva $(p<.05)$. Se mostraron correlaciones positivas de magnitud media entre el peso en hombres y los niveles de AC $(R h o=.617 ; p<.01)$. Sólo se hallaron correlaciones significativas entre los niveles de ansiedad motora y el puesto obtenido en competición en el grupo masculino ( $r h o=-.635 ; p<.05)$, de forma que a mejor puesto $\left(1^{\circ}, 2^{\circ}\right.$ o $3^{\circ}$ ), fue más probable que existieran mayores niveles de ansiedad motora.

\section{Discusión y conclusiones}

Los judokas evaluados presentaron menores niveles de ansiedad en los tres factores que la muestra de futbolistas sénior (García-Naveira \& Ruiz-Barquín, 2015). Los resultados mostraron la importancia de considerar la categoría por sexo, la categoría de peso y el nivel de rendimiento obtenido a la hora de evaluar la ansiedad, si bien estos resultados se dieron exclusivamente en el grupo de hombres. Por tanto, los resultados confirman la necesidad de realizar intervenciones psicológicas diferenciales considerando las variables sexo e incluso categoría de peso (Ruiz, 2005). Los menores niveles de ansiedad obtenidos en judokas, en comparación a otros deportistas, deben ser tomados con cautela, dado que se está comparando cuerpos militares y de seguridad con muestras civiles. La ausencia de resultados significativos obtenidos en el grupo de judokas mujeres podría ser debido a un reducido número muestral $(n=10)$, debiéndose incrementar en posteriores estudios el número de participantes en ambos sexos.

\section{Referencias}

Andrade E.M., Lois, G., \& Arce, C. (2007). Propiedades psicométricas de la versión española del Inventario de Ansiedad Competitiva CSAI-2R en deportistas. Psicothema, 19(1), 150-155.

García-Naveira, A., \& Ruiz-Barquín, R. (2015). Adaptación del ISRA-B a la población deportiva a través de jugadores de fútbol de rendimiento. Ansiedad y Estrés, 21(1), 83-94.

Martens, R., Vealey, R. S., \& Burton, D. (1990). Competitive anxiety in sport. Champaign, Il: Human Kinetics.

Miguel-Tobal, J.J., \& Cano-Vindel, A.R. (2002). ISRA. Inventario de Situaciones y Respuestas de Ansiedad (5 ${ }^{\text {a }}$ Edición).Madrid: TEA Ediciones.

Montero, C., Moreno, González, I., Pulido, J. J., \& Cervelló, E. M. (2012). Ansiedad estado precompetitiva en judocas. Revista de Artes Marciales Asiáticas, 7(1), 26-43. doi: 10.18002/rama.v8i2.977

Ruiz, R. (2005). Análisis de las diferencias de personalidad en el deporte del judo a nivel competitivo en función de la variable sexo y categoría de edad deportiva. Cuadernos de psicología del deporte, 5(1-2), 29-48.

Ruiz, R. (2009). Evaluación de los Estados de Ánimo, Autoconfianza y Ansiedad Precompetitivos en Judokas de Alto Nivel participantes en el 32 Campeonato Nacional Militar de Judo 2008. Actas del XII Congreso Andaluz de Psicología de la Actividad Física y el Deporte. Jaén.

Simon, J. A., \& Martens, R. (1977). SCAT as predictor of A-states in varying competitive situations. In D. M. Landers \& R. W. Christina (Eds.), Psychology of motor behaviour and sport (vol. 2, pp. 146-156). Champaign, IL: Human Kinetics.

Spielberger, C. D., Gorsuch, R. L., \& Lushene, R. E. (1968). State-Trait Anxiety Inventory (STAI): Test Manual forForm X. Palo Alto, Calif.: Consulting Psychologists Press. 